\title{
INPPO actions and recognition as a driving force for progress in plant proteomics: Change of guard, INPPO update, and upcoming activities
}

Ganesh Kumar Agrawal, Dominique Job, Thomas Kieselbach, Bronwyn J. Barkla, Sixue Chen, Renu Deswal, Sabine Lüthje, Ramesh Sundar Amalraj, Georgia Tanou, Bongani Kaiser Ndimba, Rainer Cramer, Wolfram Weckwerth, Stefanie Wienkoop, Michael J. Dunn, Sun Tae Kim, Yochiro Fukao, Masami Yonekura, Lello Zolla, Jai Singh Rohila, Rungaroon Waditee-Sirisattha, Antonio Masi, Tai Wang, Abhijit Sarkar, Raj Agrawal, Jenny Renaut and Randeep Rakwal

\begin{abstract}
The International Plant Proteomics Organization (INPPO) is a non-profit organization whose members are scientists involved or interested in plant proteomics. Since the publication of the first INPPO highlights in 2012, continued progress on many of the organization's mandates/goals has been achieved. Two major events are emphasized in this second INPPO highlights. First, the change of guard at the top, passing of the baton from Dominique Job, INPPO founding President to Ganesh Kumar Agrawal as the incoming President. Ganesh K. Agrawal, along with Dominique Job and Randeep Rakwal initiated the INPPO. Second, the most recent INPPO achievements and future targets, mainly the organization of first the INPPO World Congress in 2014, tentatively planned for Hamburg (Germany), are mentioned.
\end{abstract}

\section{Change of INPPO guard}

\subsection{First INPPO President: Achievements and message from Dominique Job}

Following the foundation of INPPO together with Randeep Rakwal and Ganesh K. Agrawal (Fig. 1), I immediately spent several months to build the network, mainly by contacting colleagues representing laboratories/scientific communities worldwide, presenting them the INPPO viewpoints [1] and trying to convince them to join this novel initiative. This journey was a long but pleasant endeavor of which results were extremely promising. I was indeed very much impressed to observe the very positive attitude of the plant proteomics community toward INPPO, as we soon arrived to an incredible number of more than 650 members from many different countries in all continents. Thanks to all members for their interest in this initiative. I also participated to structure the organization (status and committees). Yet my main action was in communicating about INPPO: during international conferences, or as a guest 
Editor, e.g., together with Paul Haynes and Michel Zivy, for the first special issue on plant proteomics ever published, in 2011, in the journal PROTEOMICS [2].

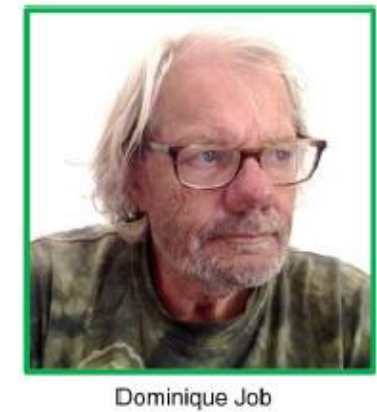

(Founding President; till July 2013)

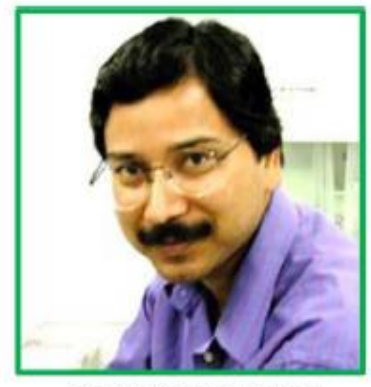

Ganesh Kumar Agrawal

(Second President; w.e.f. August 2013)

Figure 1. Change of guard at INPPO. It is the right time to be involved in plant proteomics. INPPO - by the researchers for the researchers.

This action resulted in a strong and successful collaboration between INPPO and PROTEOMICS, of which a main result was the kind offer of Wiley to regularly publish INPPO highlights, as the PROTEOMICS journal has been doing for the HUPO (Human Proteome Organization). I am glad to see that a second special issue has been released in this Journal, thanks to the Guest Editors Rainer Cramer, Laurence Bindschedler and Ganesh K. Agrawal [3]. I have also been very impressed by several important actions for promoting INPPO, among which stand out the creation of our website thanks to Raj Agrawal and Abhijit Sarkar, the publication of our superb and well-documented INPPO Newsletters (http://www.inppo.com/newsletter.jsp) thanks to the tremendous and very professional work done by Bongani Ndimba, Georgia Tanou and Abhijit Sarkar, and the strong implication of a group of colleagues in education and training under the leadership of Thomas Kieselbach.

I have been very pleased and honored to serve as the first INPPO President. However, I think responsibilities should rotate so that everyone can make different and complementary views toward the success of this organization. For the next future, my main message will be to start collaborative projects in plant proteomics at the levels of countries and/or continents both on fundamental and applied perspectives. One way to achieve this will be to further develop collaborations with existing networks, notably MASCP (the proteomics subcommittee of the Multinational Arabidopsis Steering Committee), iMOP (the HUPO initiative on Model Organism Proteomes) and HUPO. This activity will allow us not only to better understand the unique plant system (plants are sessile organisms) but also help create new plant varieties and crops that are required to feed an ever increasing world population, to improve our environment, and to promote novel useful biotechnologies (e.g., plant factories for novel biomaterials, vaccines, and bioenergy). Plant proteomics is a very dynamic discipline. A search using the terms "plant proteomics" on the Web of Science identifies as of August 2013 a total of 6356 publications compared to only 3769 at the end of 2010. 
INPPO will now continue its development under the leadership of Ganesh K. Agrawal, the new INPPO President, to whom I wish the largest success with my full support. One major future action will be holding the first INPPO World Congress 2014, which will be in addition to scientific aspects, an opportunity to strengthen our organization, notably concerning the election of INPPO members to key INPPO positions and the creation of a forum for discussions toward deciphering further scientific and structuring roles of INPPO.

Being President of an organization such as INPPO cannot be viewed as a solo endeavor. It is a great pleasure to express all my thanks to all colleagues and INPPO members who fuelled the development of the organization. I wish a long life to INPPO.

\subsection{Newly appointed second INPPO President: Message from Ganesh K. Agrawal}

It is an honor to be appointed as the INPPO President following the tenure of Dominique Job and with this responsibility come new challenges to be accomplished hereafter (Fig. 1). I would like to thank all the INPPO founding members, members, and the dedicated INPPO supporting staff - Abhijit Sarkar and Raj Agrawal for their continuous and selfless contributions for bringing INPPO to the present day. My message to the INPPO members and the other communities involved in science-related works is very simple: To give a new dimension in line with what has been proposed in the INPPO viewpoint [1]. One way is to accelerate the process of involving each and every plant proteomics researcher into the INPPO vision. The INPPO webpage serves as a great resource providing constantly updated information on various INPPO activities not only to the INPPO members and also to the scientific community in general.

The top priorities in the next few years will be: (i) to raise funds for INPPO to run it more effectively. At the moment, supporting staffs are volunteering their valuable time but we would like to create positions in which staffs receive an appropriate amount of financial support; (ii) to organize the first INPPO World Congress in the year 2014. As aforementioned by Dominique Job, this Congress would provide a forum bringing together INPPO members from around the world to share their research and teaching resources in an open and productive meeting; (iii) to expand INPPO chapters to most of the member countries; (iv) to drive the importance of plant-based food and proteomics application therein; (v) to continue the publication of the annual (or biannual) INPPO highlights that summarizes the recent news from INPPO and includes updates from individual member countries; and (vi) to continue highlighting the importance and advances in plant proteomics via regular special issues on specific topics, in the journal PROTEOMICS.

As incoming President, I would like to emphasize one point to all INPPO members. Although the position in the INPPO organizational structure is important, one's 
focus must be to contribute effectively by taking initiatives to organization progress in plant proteomics, independently or collectively. Therefore, it is my sincere request to the INPPO members and the organization body to further reinforce their involvement in this endeavor. Without your help and support, INPPO could not come to stand on the world stage as a respected organization devoted to promoting plant proteomics. The take-home message is - Do for others as you will do for yourself, especially to the next generation by teaching and disseminating plant proteomics knowledge.

\section{INPPO achievements and future works in promoting plant proteomics, collaborations, and the first world congress in 2014}

The achievements till 2011 were mentioned in the first INPPO highlights [4]. Since then (to July 2013), the progress in terms of membership has been slower compared to initial boost that was achieved by 2011 reflecting the fact that INPPO has now attracted a major part of the international plant proteomics community. At present, the INPPO members have increased further to 666. The INPPO country representatives are now 76, representing 42 countries and one organization (The International Rice Research Institute, IRRI, http://irri.org). Within two years, between the $17^{\text {th }}$ August 2011 and $17^{\text {th }}$ August 2013, INPPO website received 8524 visitors from across the globe (http://www.inppo.com). To keep members aware of the INPPO activities and also to give a chance for members to discuss their research and related activities, the Newsletter section was initiated in 2012 (http://www.inppo.com/newsletter.jsp).

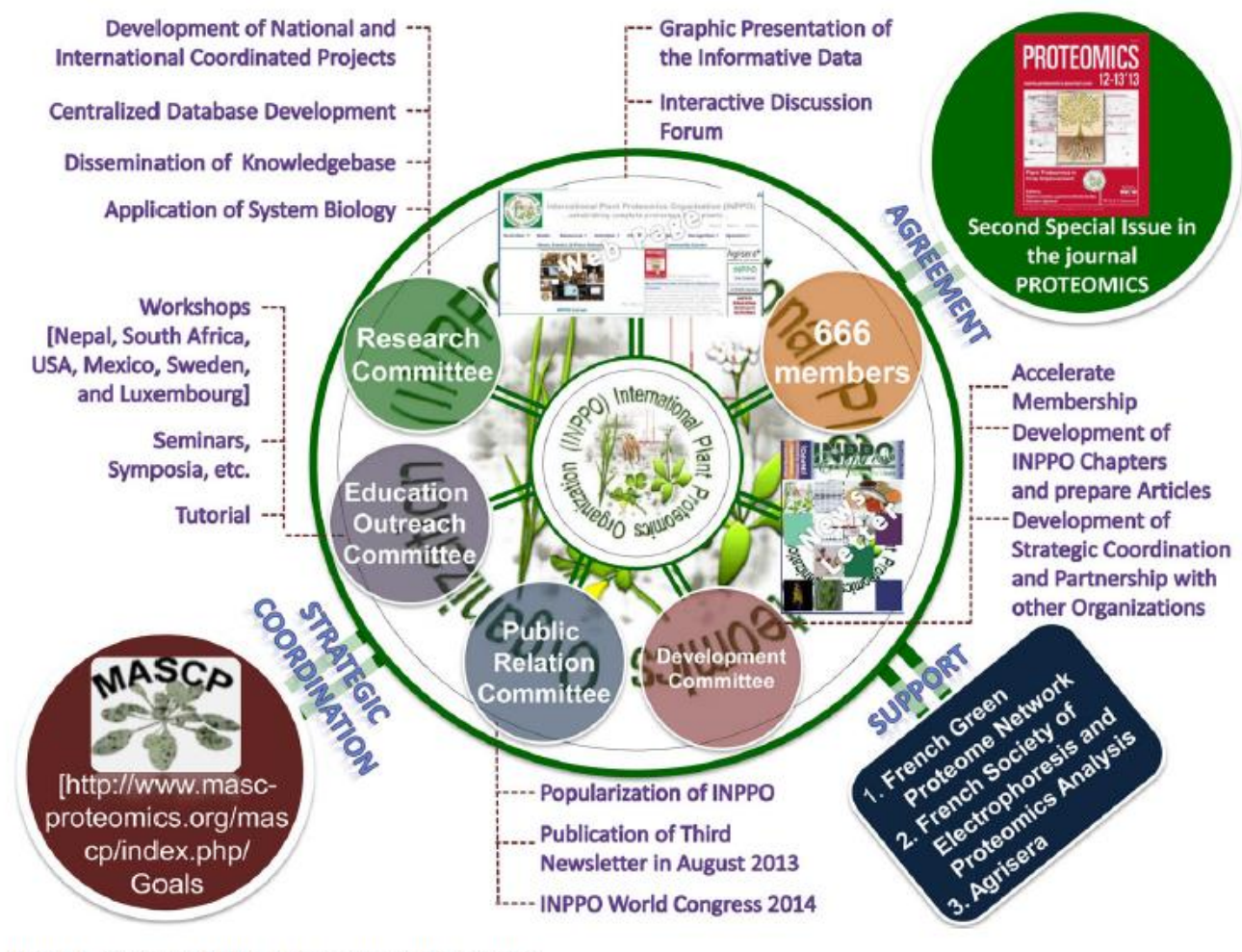

Figure 2. Update on INPPO achievements and projections. 
The third and latest issue can be accessed at http://www.inppo.com/newsletter/INPPO_Express_2013_\%20vol\%202_Issue_1.pdf. These Newsletters are intended to profile influential scientists and publish information and popular articles. The intention is to publish two issues per annum but the Editors depend on the submissions - and the Editorial team tries to publish all the received "news and views". The INPPO Core Committee is encouraging the members to continue their support by actively taking part in the Newsletter publication (Fig. 2).

In 2012, the CHAPTERS series (i.e., plant proteomics sub-organizations in respective member countries) was conceived to highlight the status of plant proteomics worldwide. The first chapter established formally was the INPPOIndia-Nepal. In this context, an article has been published on the current situation (issues, resources, people, and research) in India and Nepal [5]. Five other chapters (INPPO-China, INPPO-Japan, INPPO-South Africa, INPPO-South Korea, and INPPOThailand) are in the pipeline. In parallel, in France, an equivalent initiative has been conducted since many years - The Green Proteomic Network - gathering together a number of public institutions (INRA, CNRS) and several universities, as well as more than 80 scientists. Michel Zivy leads this network, which meets once a year with the principal aim of structuring national projects in plant proteomics (under the umbrella of INPPO) and sharing its resources and equipments. Simultaneously, articles highlighting the status of plant proteomics research are also being presented. For example, the plant proteomics status in South Korea was reviewed [6]. Tai Wang in China is currently coordinating with the other CRs for China to write such an article. Discussion with the CRs in other countries (e.g., Greece, Cyprus, and Mexico) is also in progress for initiating the composition of such articles. The aim of these review articles is to help bring plant proteomics research status (and challenges) in each country onto a platform for dissemination and discussion therein. Such activity is critical to the INPPO goal of taking proteomics knowledge to each and every laboratory working on plants, and will further help increase the membership of INPPO.

The first special issue of plant proteomics was published in the PROTEOMICS journal in May 2011 (see contents at - http://onlinelibrary.wiley.com/doi/10.1002/ pmic.v11.9/issuetoc) [2]. About two years later this issue has been cited more than 170 times according to Web of Science, testifying the wide interest and visibility of our discipline. The second special issue of plant proteomics, with a focus on crop improvement, has also been successfully completed in June 2013 (see contents at http://onlinelibrary.wiley.com/doi/10.1002/pmic.v13.12-13/issuetoc) [3]. The continuous support and collaboration of the PROTEOMICS journal with INPPO is greatly appreciated. The next and third special issue on plant proteomics is targeted for 2015. To further popularize and disseminate information on plant proteomics in different countries, it would be worthwhile to publish, independently of the annual appearance of the INPPO highlights, an update of plant proteomics in respective

\section{http://repository.uwc.ac.za}


countries as highlights in the journal PROTEOMICS. In parallel, in the years 2012 and 2013, several INPPO members worked together and published review articles on hot topics in other relevant proteomic journals. A first such review on the subject translational plant proteomics - was published in the Journal of Proteomics [7], followed by two reviews focusing on the application and translational aspects of plant proteomics in the new fields of nanoproteomics [8] and biofuel crops [9]. Another comprehensive review with particular focus towards plant proteomics application in food security and safety issues was published in Mass Spectrometry Reviews [10].

INPPO action toward increased collaboration (bench-work/wet-lab) for both new and established researchers has also been initiated. Scientific missions for students and young researchers have been developed for the past few years aiming to interact with established proteomics laboratories, to learn and implement plant proteomics research, which would be difficult otherwise due to the lack of facilities and high-end equipment including the experience of handling such equipment, at their respective laboratories/countries. This type of educational initiative among the INPPO members is important and it is desirable to see such activity, support, and exchange of knowledge increase in the future. Such creativity will bring innovation and changes in plant proteomics at the ground level. Here, we would like to thank INPPO members who have contributed to these first collaborations under the INPPO umbrella. Masami Yonekura (Japan) has accepted Carlo F. Moro (Brazil) as a Ph.D. student to work on Lotus seed proteomics along with Lello Zolla (CR from Italy) and Yoichiro Fukao (CR from Japan); Yoichiro Fukao is working with Sajad M. Zargar (India) for training him in advanced proteomics technologies, especially gel-free proteomics approaches; Bongani Ndimba (CR from South Africa) for hosting two post-doctoral fellows Ramaschandra Sharathchandra (India) and Omodele Ibraheem (Nigeria); Jai Singh Rohila (USA) for hosting a South Korean researcher Dea-Wook Kim on wheat seed germination proteome analysis using gel-based and gel-free approaches; and Antonio Masi (Italy) has accepted a first-year Ph.D. candidate (Leonard Barnabas at Sugarcane Breeding Institute, India) for conducting the plant-pathogen proteomics research in his laboratory. It is noted that this list most probably is not complete and that there would be more examples to add. Even if they are not mentioned they are also greatly appreciated. These examples greatly underscore the collaborative spirit of the INPPO members and their dedication to teaching. We hope these examples will be followed around the world.

Much progress has also been made in the work of the Education Outreach Committee. The committee released the document "TEN THINGS you need to know about INPPO” (General information, Goals, Organization, Initial work) to provide an easy way of disseminating the INPPO message to help increase the number of INPPO members. At present, this document is available in eleven languages (English, French, German, Greek, Italian, Japanese, Korean, Polish, Spanish, Swedish and Thai) and is publicly accessible on the INPPO website (http://www.inppo.com/tenthings.jsp). Our goal is to have this document in as many 
languages as possible; the Education Outreach Committee requests the help of all the CRs to assist in this process. The initial goals of the Educational Outreach Committee focused on organizing INPPO seminars, workshops, and mini-symposia on plant proteomics. In 2012, this work included numerous events at universities and plant biology conferences in the USA and different European, Asian, and African countries (see Activities > Education Outreach at www.inppo.com). Examples include, for instance, an "Omics" workshop for high school teachers at the University of Florida, Gainesville (USA), a one-day high school workshop entitled "proteins to proteomics: introductory crash course" at Maharishi Valmiki Higher Secondary School, Gaur (Nepal), a one-day proteomics and mass spectrometry seminar at the Department of Botany, University of Delhi (India), and the seminar day "Making Sense of the Complexities of Proteomics Data" at the Centre for Proteomic and Genomic Research (CPGR) in Cape Town (South Africa). In addition, INPPO workshops were held the ${ }_{23}{ }^{\text {rd }}$ International Conference on Arabidopsis Research in Vienna (Austria), the 2012 Plant Biology Conference in Austin (USA), the Proteomelux conference in Luxemburg (Luxemburg) and the Scandinavian Plant Proteomics Day in Umea (Sweden). The activities of 2012 were followed by a quiet period in the beginning of this year, but the Educational Outreach Committee will continue their work to organize educational events in plant proteomics in the future. A long-term goal is to establish regular INPPO courses on proteomics, offering graduate students an opportunity to learn basic and advanced proteomics techniques using practical applications and how to address biological questions in plant biology.

Last but not the least, the Research Committee has also been discussing various research topics and these are still under consideration among the respective members. For example, in the past months the development of an equivalent to MASCP Gator (Arabidopsis; http://gator.masc-proteomics.org) for rice has been proposed. Contacts are established with MASCP (Josh Heazlewood) toward this end. One major output of the discussion among the members of the Research Committee is organizing the first INPPO World Congress 2014 in Hamburg, Germany, which will be headed by Sabine Lüthje (CR from Germany). Why to organize an international plant proteomic congress? This is because of the central role of plants in the provision of food and countless molecules and biomaterials for humanity. Therefore plant sessions are established at several international proteome conferences related to the HUPO and to other organizations, and participation of plant scientists to these conferences is important and has to be pursued. However, besides such transversal actions the INPPO congress is to provide a forum for discussion and for the effective exchange of information and new ideas on every aspect of plant proteomics. Besides plenary lectures and invited speakers, poster sessions will play a central role. Talks will be selected mainly from submitted abstracts. Additionally, meetings of INPPO committees and members gathering are planned. Organizers aim to give ample opportunity for everyone especially for early career scientists to participate in this congress. The venue will be at the main campus of the University of Hamburg in the center of the city, near the central station and with direct connection to the airport. The planned time 
schedule of the congress is four days in early September 2014. So far the scientific committee is installed and the organizing committee is ready to go. First circular will be announced as soon as topics are decided and invited speakers are confirmed. We are still in a very early stage of planning, but we would like to facilitate your scheduling by announcing locality, date and times very early. The INPPO members and scientific community will be informed of the next steps toward this gathering of plant proteomers via the University of Hamburg (domain: INPPO2014.uni-hamburg.de, email: INPPO2014@uni-hamburg.de) and the INPPO website (www.inppo.com) and invitations will be issued therein.

\section{Concluding remarks}

INPPO is both a working group in science and an instrument to popularize plant proteomics and to convince funding agencies and/or private foundations to support projects in plant proteomics. The highlighted achievements and future targets in the rest of 2013 and onwards are challenges and that will be addressed by the INPPO scientific committees. However, original ideas and suggestions for activities are welcomed from individual members who can freely contact and discuss these with the respective committees. The direction INPPO takes from now on is critical. INPPO is committed to work together with greater vigor, notably toward the establishment of joint proteomics projects in model plants and crops. This is a difficult task that requires a fully integrated international effort in which INPPO aims to be a coordinating partner. INPPO once again emphasizes that it exists as it is today due to the great support of the members and the hard work of their committees.

The authors acknowledge all the INPPO members for their strong support in the building up of a global platform for plant proteomics and their active involvement in various ongoing INPPO activities. The authors also thank the scientific and non-scientific communities for their direct and indirect supports. INPPO is highly grateful to PROTEOMICS and in particular, Dr. Hans-Joachim Kraus, Senior Publishing Editor, Wiley-VCH Verlag, Weinheim (Germany) for generous support in making possible the INPPO highlights.

The authors have declared no conflict of interest. 


\section{References}

[1] Agrawal, G. K., Job, D., Zivy, M., Agrawal, V. P. et al., Time to articulate a vision for the future of plant proteomics - A global perspective: An initiative for establishing the International Plant Proteomics Organization (INPPO). Proteomics 2011, 11, 1559-1568.

[2] Job, D., Haynes, P. A., Zivy, M., Plant proteomics. Proteomics 2011, 11, 1557-1558. [3] Cramer, R., Bindschedler, L., Agrawal, G. K., Plant proteomics in crop improvement.

Proteomics 2013, 12-13, 1771.

[4] Agrawal, G. K., Sarkar, A., Agrawal, R., Ndimba, B. et al., Boosting the globalization of plant proteomics through INPPO: Current developments and future prospects. Proteomics 2012, 12, 359-368.

[5] Deswal, R., Gupta, R., Dogra, V., Singh, R. et al., Plant proteomics in India and Nepal: Current status and challenges ahead. Physiol. Mol. Biol. Plants 2013. DOI 10.1007/s12298-013-0198-y.

[6] Lee, S.-J., Kang, K. Y., Jwa, N.-S., Kim, D.-W. et al., A decade of plant proteomics in South Korea: The international plant proteomics organization (INPPO) perspective and involvement. Plant Omics Journal 2012, 5, 458-465.

[7] Agrawal, G. K., Pedreschi, R., Barkla, B. J., Bindschedler, L. V. et al., Translational plant proteomics: A perspective. J. Proteomics 2012, 75, 4588-4601.

[8] Agrawal, G. K., Timperio, A. M., Zolla, L., Bansal, V. et al., Biomarker discovery and applications for foods and beverages: Proteomics to nanoproteomics. J. Proteomics 2013, doi:10.1016/j.jprot.2013.04.014.

[9] Ndimba, B. K., Ndimba, R. J., Johnson, T. S., Waditee-Sirisattha, R. et al., Biofuels as a sustainable energy source: An update of the applications of proteomics in bioenergy crops and algae. J.Proteomics 2013, doi:10.1016/j.jprot.2013.05.041.

[10] Agrawal, G. K., Sarkar, A., Righetti, P. G., Pedreschi, R. et al., A decade of plant proteomics and mass spectrometry: Translation of technical advancements to food security and safety issues. Mass Spectrometry Reviews 2013, 32, 335-365.

\section{Addendum}

2GRADE Academy Private Limited, Adarsh Nagar, Birgunj, Nepal

3CNRS/UCBL/INSA/Bayer CropScience Joint Laboratory, UMR 5240, Bayer CropScience, Lyon, France

4Department of Chemistry, Umea University, Umea , Sweden

5Instituto de Biotecnologia, Universidad Nacional Autonoma de Mexico, Morelos, Mexico

${ }^{6}$ Department of Biology, Interdisciplinary Center for Biotechnology Research (ICBR), Cancer \& Genetics Research Complex, University of Florida, Gainesville, FL, USA

7Molecular Plant Physiology and Proteomics Laboratory, Department of Botany, University of Delhi, Delhi, India ${ }^{8}$ Oxidative Stress and Plant Proteomics Group, University of Hamburg, Biocenter Klein Flottbek, Hamburg, Germany

9Plant Pathology Section, Sugarcane Breeding Institute, Indian Council of Agricultural Research, Tamil Nadu, India

\section{http://repository.uwc.ac.za}


10Faculty of Agriculture, Aristotle University of Thessalonki, Thessaloniki, Greece ${ }^{11}$ Proteomics Research and Services Unit, Agricultural Research Council, InfruitecNietvoorbij Campus, Stellenbosch, South Africa

${ }^{12}$ Proteomics Research Group, Department of Biotechnology, University of the Western Cape, Bellville, South

Africa

13Department of Chemistry, University of Reading, Reading, United Kingdom

14Molecular Systems Biology, University of Vienna, Vienna, Austria

15UCD Conway Institute of Biomolecular and Biomedical Research, School of Medicine and Medical Science, University College Dublin, Dublin, Ireland

16Department of Plant Bioscience, Pusan National University, Miryang, South Korea

17Graduate School of Biological Sciences, Nara Institute of Science and Technology, Ikoma, Japan

18Plant Global Educational Project, Nara Institute of Science and Technology, Ikoma, Japan

19Laboratory of Molecular Food Functionality, College of Agriculture, Ami, Ibaraki, Japan

${ }^{20}$ Department of Ecology and Biology, University Tuscia, Piazzale Universita, Viterbo, Italy

${ }^{21}$ Department of Biology and Microbiology, South Dakota State University, Brookings, SD, USA 22Department of Microbiology, Faculty of Science, Chulalongkorn University, Bangkok, Thailand 23DAFNAE, University of Padova, Legnaro, Italy

24Key Laboratory of Plant Molecular Physiology, Institute of Botany, Chinese Academy of Sciences, Xiangshan, Haidianqu, Beijing, China

25International Plant Proteomics Organization (INPPO www.inppo.com)

26Institute of Genetic Medicine and Genomic Science (IGMGS), Badu, Kolkata, West Bengal, India

27Centre de Recherche Public-Gabriel Lippman, Department of Environment and Agrobiotechnologies (EVA), Belvaux, GD, Luxembourg

${ }^{28}$ Department of Anatomy I, School of Medicine, Showa University, Shinagawa, T kyo, Japan

29Organization for Educational Initiatives, University of Tsukuba, Tsukuba, Japan 